\title{
A simple commutativity condition for block decimators and expanders
}

\author{
Didier Pinchon and Pierre Siohan
}

\begin{abstract}
Commutativity rules play an essential role when building multirate signal processing systems. In this letter, we focus on the interchangeability of block decimators and expanders. We, formally, prove that commutativity between these two operators is possible if and only if the data blocks are of an equal length corresponding to the greatest common divisor of the integer decimation and expansion factors.
\end{abstract}

\section{Index Terms}

Block sampling, Decimation, Expansion, Commutativity.

\section{INTRODUCTION}

The polyphase decomposition, introduced by Bellanger et al. in 1976 [1], is a key element in multirate signal processing to reduce the computational complexity of digital equipments. Its implementation involves decimation and expansion operators. For an input sequence $x[n]$, a conventional decimator, with integer decimation factor $p_{1}$, only retains the samples at time multiple of $p_{1}$. A conventional expander, with integer expansion factor $p_{2}$, inserts $p_{2}-1$ samples between each pair of consecutive $x[n]$ samples. It is well-known that conventional decimators and expanders can commute if and only if $p_{1}$ and $p_{2}$ are coprime. The important question of commutativity of these basic operators also occurs when designing either multidimensional [2], [3] or block processing systems [4], [5]. Block samplers are of a particular interest to deal with the class of incompatible nonuniform filter banks [6]. Block decimators (resp. expanders) are defined by parameters that, in addition to the decimation (resp. expansion) factors, include the block length. In [5] the authors consider a block decimator $\downarrow\left(q_{1}, p_{1}\right)$ and an expander $\uparrow\left(q_{2}, p_{2}\right)$, with $q_{1}, q_{2}$ the block sizes and $p_{1}, p_{2}$ the decimation and expansion factors, respectively. Assuming $q_{1}$ and $q_{2}$ are not necessarily equal and $p_{1}, p_{2}$ non necessarily integers, they give three joint conditions that are necessary and sufficient conditions to insure the commutativity of $\downarrow\left(q_{1}, p_{1}\right)$ and $\uparrow\left(q_{2}, p_{2}\right)$. However no examples are given with unequal block lengths and/or rational non integer sampling ratios where the three conditions are satisfied. In [4], the commutativity of up and down sampling is studied when the sampling ratios are integers but with unequal block lengths. Again, the authors do not provide any example where the commutativity is obtained with unequal block lengths.

Our notations slightly differ from those in [5]. For integers $q, p$ such that $1 \leq q<p$, let us denote by $D(q, p)$ the decimator with block length $q$ and sampling ratio $p / q$. Such a decimator receives a sequence of $p$ consecutive input symbols of a signal, keeps the first $q$ ones and discards the last $p-q$ symbols. For integers $q, p$ such that $1 \leq q<p, E(q, p)$ denotes the expander with block length $q$ and sampling ratio $p / q$ : each block with length $q$ of the input signal is transmitted with the addition of $p-q$ zero taps.

In this letter, the following theorem is proved.

Theorem. Let $q_{1}, p_{1}, q_{2}, p_{2}$ be integers such that $1 \leq q_{1}<p_{1}$ and $1 \leq q_{2}<p_{2}$. Then $D\left(q_{1}, p_{1}\right)$ and $E\left(q_{2}, p_{2}\right)$ commute if and only if $q_{1}=q_{2}=\operatorname{gcd}\left(p_{1}, p_{2}\right)$.

For $q_{1}=q_{2}=1$, it is already well known that, when $q_{1}=q_{2}=\operatorname{gcd}\left(p_{1}, p_{2}\right)$, then $D\left(q_{1}, p_{1}\right)$ and $E\left(q_{2}, p_{2}\right)$ commute (see for example [7], pages 119 and 179). The extension of this result to arbitrary equal block lengths

D. Pinchon is with the Institute of Mathematics, University Paul Sabatier, 118, route de Narbonne, 31062 Toulouse Cedex, France. E-mail: didier.pinchon@math.univ-toulouse.fr

* P. Siohan, corresponding author (Ph.: +33 2991243 05, Fax: +33 2991240 98, Email: pierre.siohan@ orange.com), is with Orange Labs, 4, rue du Clos Courtel, 35512 Cesson-Sévigné, France. 
corresponds to the easiest part of our theorem. However, as we could not find any proof of it, in order to provide a self contained paper, we demonstrate it in our Lemmas 2 and 3.

\section{PROOF OF THE THEOREM}

Before proving the theorem, let us introduce some notations and definitions.

For $n \in \mathbb{Z}$ and $m>0$ two integers, the quotient $a$ and the remainder $b$ of the euclidean division of $n$ by $m$ are denoted by $a=q u o(n, m), b=\operatorname{rem}(n, m)$, which may be also written in a condensed form $(a, b)=\operatorname{div}(n, m)$.

Let $1 \leq q_{1}<p_{1}$. Applied to a discrete-time input signal, named in short a sequence, $x=(x[n], n \in \mathbb{Z})$, the decimator $D\left(q_{1}, p_{1}\right)$ returns an output sequence $t=(t[k], k \in \mathbb{Z})$ obtained by

$$
\begin{aligned}
& (a, b)=\operatorname{div}\left(n, p_{1}\right),\left(a \in \mathbb{Z}, 0 \leq b<p_{1}\right), \\
& k=a q_{1}+b, \text { if } b<q_{1}, \\
& t[k]=x[n]
\end{aligned}
$$

When $q_{1} \leq b<p_{1}$, the $x[n]$ sample is discarded.

For $1 \leq q_{2}<p_{2}$, the expander $E\left(q_{2}, p_{2}\right)$ is now applied to the input sequence $t=(t[k], k \in \mathbb{Z})$, producing a sequence $y_{1}=\left(y_{1}[m], m \in \mathbb{Z}\right)$ defined by

$$
\begin{aligned}
& (\alpha, \beta)=\operatorname{div}\left(k, q_{2}\right),\left(\alpha \in \mathbb{Z}, 0 \leq \beta<q_{2}\right) \\
& y_{1}\left[\alpha p_{2}+\beta\right]=t[k] .
\end{aligned}
$$

and the unassigned symbols in the sequence $y_{1}=\left(y_{1}[m], m \in \mathbb{Z}\right)$ are set to zero. The transformation to go from $x=(x[n], n \in \mathbb{Z})$ to $y_{1}=\left(y_{1}[m], m \in \mathbb{Z}\right)$ is denoted by $E\left(q_{2}, p_{2}\right) D\left(q_{1}, p_{1}\right)$.

Equations (1)-(5) allow us to define a function $f_{1}\left(q_{1}, p_{1}, q_{2}, p_{2} ; \bullet\right)$, depending on parameters $q_{1}, p_{1}, q_{2}, p_{2}$, and defined on integers $n \in \mathbb{Z}$ that are expressed in an intuitive algorithmic language in (6) such that, when $f_{1}\left(q_{1}, p_{1}, q_{2}, p_{2} ; n\right) \neq-\infty, y_{1}\left[f_{1}\left(q_{1}, p_{1}, q_{2}, p_{2} ; n\right)\right]=x[n]$. When $f_{1}\left(q_{1}, p_{1}, q_{2}, p_{2} ; n\right)=-\infty$, the symbol $x[n]$ is discarded and $y_{1}[m]=0$ when $m \in \mathbb{Z}$ is not in the image of $f_{1}\left(q_{1}, p_{1}, q_{2}, p_{2} ; \bullet\right)$.

$$
\left[\begin{array}{c}
f_{1}=\operatorname{proc}\left(q_{1}, p_{1}, q_{2}, p_{2} ; n\right) \\
\text { local } a, b, \alpha, \beta \\
(a, b)=\operatorname{div}\left(n, p_{1}\right) \\
\text { if } b<q_{1} \text { then } \\
(\alpha, \beta)=\operatorname{div}\left(a q_{1}+b, q_{2}\right) \\
\text { return } \alpha p_{2}+\beta \\
\text { else return }-\infty \\
\text { end }
\end{array}\right.
$$
by

The action of $E\left(q_{2}, p_{2}\right)$ on the input sequence $x=(x[n], n \in \mathbb{Z})$ produces a sequence $z=(z[l], l \in \mathbb{Z})$ defined

$$
\begin{aligned}
& (c, d)=\operatorname{div}\left(n, q_{2}\right)\left(c \in \mathbb{Z}, 0 \leq d<q_{2}\right), \\
& l=c p_{2}+d, \\
& z[l]=x[n] .
\end{aligned}
$$

Then, applying the decimator $D\left(q_{1}, p_{1}\right)$ to the input sequence $z=(z[l], l \in \mathbb{Z})$ produces the sequence $y_{2}=$ $\left(y_{2}[m], m \in \mathbb{Z}\right)$, such that

$$
\begin{aligned}
& (\gamma, \delta)=\operatorname{div}\left(l, p_{1}\right)\left(\gamma \in \mathbb{Z}, 0 \leq \delta<p_{1}\right), \\
& y_{2}\left[\gamma q_{1}+\delta\right]=z[l], \text { if } \delta<q_{1} .
\end{aligned}
$$

Again unassigned samples in the sequence $y_{2}=\left(y_{2}[m], m \in \mathbb{Z}\right)$ are set to zero. The overall transformation is denoted by $D\left(q_{1}, p_{1}\right) E\left(q_{2}, p_{2}\right)$. 
In a similar way, a function $f_{2}\left(q_{1}, p_{1}, q_{2}, p_{2} ; \bullet\right)$, depending on parameters $q_{1}, p_{1}, q_{2}, p_{2}$, and defined for $n \in \mathbb{Z}$ is defined by (12). For the values of $n$ such that $f_{2}\left(q_{1}, p_{1}, q_{2}, p_{2} ; n\right) \neq-\infty$, we have $y_{2}\left[f_{2}\left(q_{1}, p_{1}, q_{2}, p_{2} ; n\right)\right]=x[n]$. When $\left.f_{2}(n)\right]=-\infty$, the symbol $x[n]$ is discarded and $y_{2}[m]=0$ when $m$ is not in the image of $f_{2}$.

$$
\left[\begin{array}{c}
f_{2}=\operatorname{proc}\left(q_{1}, p_{1}, q_{2}, p_{2} ; n\right) \\
\text { local } c, d, \gamma, \delta \\
(c, d)=\operatorname{div}\left(n, q_{2}\right) \\
(\gamma, \delta)=\operatorname{div}\left(c p_{2}+d, p_{1}\right) \\
\text { if } \delta<q_{1} \text { then } \\
\text { return } \gamma q_{1}+\delta \\
\text { else return }-\infty \\
\text { end }
\end{array}\right.
$$

Inverting equations (1)-(5) (resp. (7)-(11)) for given parameters $q_{1}, p_{1}, q_{2}, p_{2}$, we may introduce the function $g_{1}\left(q_{1}, p_{1}, q_{2}, p_{2} ; \bullet\right)$ (resp. $\left.g_{2}\left(q_{1}, p_{1}, q_{2}, p_{2} ; \bullet\right)\right)$ defined for $m \in \mathbb{Z}$ such that $y_{1}[m]=0$ when $g_{1}\left(q_{1}, p_{1}, q_{2}, p_{2} ; m\right)=$ $-\infty$ and $y_{1}[m]=x\left[g_{1}\left(q_{1}, p_{1}, q_{2}, p_{2} ; m\right)\right]$ otherwise (resp. $y_{2}[m]=0$ when $g_{2}\left(q_{1}, p_{1}, q_{2}, p_{2} ; m\right)=-\infty$ and $y_{2}[m]=$ $x\left[g_{2}\left(q_{1}, p_{1}, q_{2}, p_{2} ; m\right)\right]$ otherwise).

$$
\begin{aligned}
& {\left[g_{1}=\operatorname{proc}\left(q_{1}, p_{1}, q_{2}, p_{2} ; m\right)\right.} \\
& \text { local } \alpha, \beta, a, b \\
& (\alpha, \beta)=\operatorname{div}\left(m, p_{2}\right) \\
& \text { if } \beta<q_{2} \text { then } \\
& (a, b)=\operatorname{div}\left(\alpha q_{2}+\beta, q_{1}\right) \\
& \text { return } a p_{1}+b \\
& \text { else return }-\infty \\
& \text { end } \\
& {\left[\begin{array}{c}
g_{2}=\operatorname{proc}\left(q_{1}, p_{1}, q_{2}, p_{2} ; m\right) \\
\text { local } \gamma, \delta, c, d \\
(\gamma, \delta)=\operatorname{div}\left(m, q_{1}\right) \\
(c, d)=\operatorname{div}\left(\gamma p_{1}+\delta, p_{2}\right) \\
\text { if } d<q_{2} \text { then } \\
\text { return } c q_{2}+d \\
\text { else return }-\infty \\
\text { end }
\end{array}\right.}
\end{aligned}
$$

It is now obvious that the following properties are equivalent

- $D\left(q_{1}, p_{1}\right)$ and $E\left(q_{2}, p_{2}\right)$ commute,

- $f_{1}\left(q_{1}, p_{1}, q_{2}, p_{2} ; \bullet\right)=f_{2}\left(q_{1}, p_{1}, q_{2}, p_{2} ; \bullet\right)$,

- $g_{1}\left(q_{1}, p_{1}, q_{2}, p_{2} ; \bullet\right)=g_{2}\left(q_{1}, p_{1}, q_{2}, p_{2} ; \bullet\right)$.

This is clearly stated in Theorem 1 of [4] which amounts to say that the up and down block sampling with integer sampling ratios commute if and only if $g_{1}\left(q_{1}, p_{1}, q_{2}, p_{2} ; \bullet\right)=g_{2}\left(q_{1}, p_{1}, q_{2}, p_{2} ; \bullet\right)$.

The method of our proof to prove that $D\left(q_{1}, p_{1}\right)$ and $E\left(q_{2}, p_{2}\right)$ do not commute for a given subset of parameters $q_{1}, p_{1}, q_{2}, p_{2}$ will be to find a particular value of $n$, depending on $q_{1}, p_{1}, q_{2}, p_{2}$, such that $f_{1}\left(q_{1}, p_{1}, q_{2}, p_{2} ; n\right) \neq$ $f_{2}\left(q_{1}, p_{1}, q_{2}, p_{2} ; n\right)$ or $g_{1}\left(q_{1}, p_{1}, q_{2}, p_{2} ; n\right) \neq g_{2}\left(q_{1}, p_{1}, q_{2}, p_{2} ; n\right)$.

Notations. In a context where parameters $q_{1}, p_{1}, q_{2}, p_{2}$ are fixed, $f_{1}\left(q_{1}, p_{1}, q_{2}, p_{2} ; n\right)$ will be denoted simply by $f_{1}(n)$. In the evaluation of $f_{1}\left(q_{1}, p_{1}, q_{2}, p_{2} ; n\right)$ following (6), the value assigned to a local variable like $a$ will be denoted by $a\left(q_{1}, p_{1}, q_{2}, p_{2} ; n\right)$, but only by $a(n)$ in the context of fixed values for the parameters $q_{1}, p_{1}, q_{2}, p_{2}$, and even simply $a$ when a given fixed value of $n$ is considered. The same notation simplification will apply also for function $f_{2}$ defined by (12) and for functions $g_{1}$ and $g_{2}$ defined by (13) and (14).

The following exchange property will be useful to restrict the number of cases to study on parameters $q_{1}, p_{1}, q_{2}, p_{2}$. 
Lemma 1. Let $q_{1}, p_{1}, q_{2}, p_{2}$ be integers with $1 \leq q_{1} \leq p_{1}, 1 \leq q_{2} \leq p_{2}$. Then $D\left(q_{1}, p_{1}\right)$ and $E\left(q_{2}, p_{2}\right)$ commute if and only if $D\left(q_{2}, p_{2}\right)$ and $E\left(q_{1}, p_{1}\right)$ commute.

Proof.- For any set of parameters $q_{1}, p_{1}, q_{2}, p_{2}$, exchanging $\left(q_{1}, p_{1}\right)$ and $\left(q_{2}, p_{2}\right)$ in the definition (6) and changing the name of the local variables $(a, b, \alpha, \beta)$ by $(\alpha, \beta, a, b)$ gives the definition (13), and thus

$$
f_{1}\left(q_{1}, p_{1}, q_{2}, p_{2} ; n\right)=g_{1}\left(q_{2}, p_{2}, q_{1}, p_{1} ; n\right), n \in \mathbb{Z} .
$$

In a similar way

$$
f_{2}\left(q_{1}, p_{1}, q_{2}, p_{2} ; n\right)=g_{2}\left(q_{2}, p_{2}, q_{1}, p_{1} ; n\right), n \in \mathbb{Z} .
$$

The lemma is proved by using afterwards as a commutativity criterion the equality of functions $f_{1}$ and $f_{2}$ or the equality of $g_{1}$ and $g_{2}$.

$D\left(1, p_{1}\right)$ corresponds to the traditional decimator of factor $p_{1}$ while $E\left(1, p_{2}\right)$ is the traditional expander of factor $p_{2}$. The following lemma is a classical result reobtained using our own notations.

Lemma 2. Let $p_{1}>1$ and $p_{2}>1 . D\left(1, p_{1}\right)$ and $E\left(1, p_{2}\right)$ commute if and only if $p_{1}$ and $p_{2}$ are relatively prime integers.

Proof.- For $n \in \mathbb{Z}$, we get from (6) and (12)

- If $n$ is a multiple of $p_{1}$ i.e. $n=a p_{1}$, then $f_{1}(n)=a p_{2}$, otherwise $f_{1}(n)=-\infty$,

- If $n p_{2}$ is a multiple of $p_{1}$ i.e. $n p_{2}=\gamma p_{1}$, then $f_{2}(n)=\gamma$, otherwise $f_{2}(n)=-\infty$.

If $p_{1}$ and $p_{2}$ are not relatively primes, then $p_{1}=d p_{1}^{\prime}, p_{2}=d p_{2}^{\prime}$ with $d>1$. Choosing $n=p_{1}^{\prime}$, we get $f_{1}\left(p_{1}^{\prime}\right)=-\infty$ because $p_{1}^{\prime}$ is not multiple of $p_{1}$. But $n p_{2}=p_{1}^{\prime} d p_{2}^{\prime}=p_{2}^{\prime} p_{1}$ and thus $f_{2}\left(p_{1}^{\prime}\right)=p_{2}^{\prime}$. This proves that $f_{1} \neq f_{2}$ meaning that $D\left(1, p_{1}\right)$ and $E\left(1, p_{2}\right)$ do not commute.

If $p_{1}$ and $p_{2}$ are relatively primes, then if $n p_{2}$ is a multiple of $p_{1}$ if and only if $n$ is a multiple of $p_{1}$, and $f_{1}=f_{2}$, that is $D\left(1, p_{1}\right)$ and $E\left(1, p_{2}\right)$ commute.

The following lemma allows us to multiply the parameters $q_{1}, p_{1}, q_{2}, p_{2}$ by a same positive integer which is an already well known result.

Lemma 3. Let $q_{1}, p_{1}, q_{2}, p_{2}$ be integers with $1 \leq q_{1} \leq p_{1}, 1 \leq q_{2} \leq p_{2}$ and $d>1$ an integer. Then $D\left(q_{1}, p_{1}\right)$ and $E\left(q_{2}, p_{2}\right)$ commute if and only if $D\left(d q_{1}, d p_{1}\right)$ and $E\left(d q_{2}, d p_{2}\right)$ commute.

Proof.- For $n \in \mathbb{Z}$, define $n^{\prime} \in \mathbb{Z}$ and $0 \leq \gamma<d$ by $n=n^{\prime} d+\gamma$. In the evaluation of $f_{1}\left(q_{1}, p_{1}, q_{2}, p_{2} ; n^{\prime}\right)$, we get $n^{\prime}=a\left(q_{1}, p_{1}, q_{2}, p_{2} ; n^{\prime}\right) p_{1}+b\left(q_{1}, p_{1}, q_{2}, p_{2} ; n^{\prime}\right), a\left(q_{1}, p_{1}, q_{2}, p_{2} ; n^{\prime}\right) \in \mathbb{Z}, 0 \leq b\left(q_{1}, p_{1}, q_{2}, p_{2} ; n^{\prime}\right)<p_{1}$. So

$$
\begin{aligned}
n & =a\left(q_{1}, p_{1}, q_{2}, p_{2} ; n^{\prime}\right) d p_{1}+b\left(q_{1}, p_{1}, q_{2}, p_{2} ; n^{\prime}\right) d+\gamma, \\
& =a\left(d q_{1}, d p_{1}, d q_{2}, d p_{2} ; n\right) d p_{1}+b\left(d q_{1}, d p_{1}, d q_{2}, d p_{2} ; n\right) .
\end{aligned}
$$

As $0 \leq b\left(q_{1}, p_{1}, q_{2}, p_{2} ; n^{\prime}\right) \leq p_{1}-1$ and $0 \leq \gamma<d$, we get $d p_{1}+b\left(q_{1}, p_{1}, q_{2}, p_{2} ; n^{\prime}\right) d+\gamma<d p_{1}$, and thus

$$
\begin{aligned}
& a\left(d q_{1}, d p_{1}, d q_{2}, d p_{2} ; n\right)=a\left(q_{1}, p_{1}, q_{2}, p_{2} ; n^{\prime}\right), \\
& b\left(d q_{1}, d p_{1}, d q_{2}, d p_{2} ; n\right)=b\left(q_{1}, p_{1}, q_{2}, p_{2} ; n^{\prime}\right) d+\gamma .
\end{aligned}
$$

If $b\left(q_{1}, p_{1}, q_{2}, p_{2} ; n^{\prime}\right)<q_{1}$ then from (18)

$$
b\left(d q_{1}, d p_{1}, d q_{2}, d p_{2} ; n\right) \leq d\left(q_{1}-1\right)+\gamma<d q_{1}
$$

and, since

$$
a\left(q_{1}, p_{1}, q_{2}, p_{2} ; n^{\prime}\right) q_{1}+b\left(q_{1}, p_{1}, q_{2}, p_{2} ; n^{\prime}\right)=\alpha\left(q_{1}, p_{1}, q_{2}, p_{2} ; n^{\prime}\right) q_{2}+\beta\left(q_{1}, p_{1}, q_{2}, p_{2} ; n^{\prime}\right),
$$

with $0 \leq \beta\left(q_{1}, p_{1}, q_{2}, p_{2} ; n^{\prime}\right)<q_{2}$, from (17) and (18), we may write

$$
\begin{aligned}
& a\left(d q_{1}, d p_{1}, d q_{2}, d p_{2} ; n\right) d q_{1}+b\left(d q_{1}, d p_{1}, d q_{2}, d p_{2} ; n\right) \\
& \quad=a\left(q_{1}, p_{1}, q_{2}, p_{2} ; n^{\prime}\right) d q_{1}+b\left(q_{1}, p_{1}, q_{2}, p_{2} ; n^{\prime}\right) d+\gamma \\
& \quad=\left[\alpha\left(q_{1}, p_{1}, q_{2}, p_{2} ; n^{\prime}\right) q_{2}+\beta\left(q_{1}, p_{1}, q_{2}, p_{2} ; n^{\prime}\right)\right] d+\gamma
\end{aligned}
$$


As $\left.\beta\left(q_{1}, p_{1}, q_{2}, p_{2} ; n^{\prime}\right)\right] d+\gamma<\left(q_{2}-1\right) d+\gamma<d q_{2}$, we get from (21),

$$
\begin{aligned}
& \alpha\left(d q_{1}, d p_{1}, d q_{2}, d p_{2} ; n\right)=\alpha\left(q_{1}, p_{1}, q_{2}, p_{2} ; n^{\prime}\right), \\
& \beta\left(d q_{1}, d p_{1}, d q_{2}, d p_{2} ; n\right)=d \beta\left(q_{1}, p_{1}, q_{2}, p_{2} ; n^{\prime}\right)+\gamma
\end{aligned}
$$

from which it follows that

$$
\begin{aligned}
& f_{1}\left(d q_{1}, d p_{1}, d q_{2}, d p_{2} ; n\right)=\alpha\left(d q_{1}, d p_{1}, d q_{2}, d p_{2} ; n\right) d p_{2}+d \beta\left(q_{1}, p_{1}, q_{2}, p_{2} ; n^{\prime}\right)+\gamma \\
& f_{1}\left(d q_{1}, d p_{1}, d q_{2}, d p_{2} ; n\right)=d f_{1}\left(q_{1}, p_{1}, q_{2}, p_{2} ; n^{\prime}\right)+\gamma
\end{aligned}
$$

If $b\left(q_{1}, p_{1}, q_{2}, p_{2} ; n^{\prime}\right) \geq q_{1}$ then, from (18), $b\left(d q_{1}, d p_{1}, d q_{2}, d p_{2} ; n\right) \geq d q_{1}$ and thus $f_{1}\left(d q_{1}, d p_{1}, d q_{2}, d p_{2} ; n\right)=$ $f_{1}\left(q_{1}, p_{1}, q_{2}, p_{2} ; n^{\prime}\right)=-\infty$ and the relation (23) still holds.

In a similar way, it is easy to prove the relation

$$
f_{2}\left(d q_{1}, d p_{1}, d q_{2}, d p_{2} ; n\right)=d f_{2}\left(q_{1}, p_{1}, q_{2}, p_{2} ; n^{\prime}\right)+\gamma .
$$

Relations (23) and (24) imply that $f_{1}\left(q_{1}, p_{1}, q_{2}, p_{2} ; \bullet\right)$ and $f_{2}\left(q_{1}, p_{1}, q_{2}, p_{2} ; \bullet\right)$ are equal if and only if $f_{1}\left(d q_{1}, d p_{1}, d q_{2}, d p_{2} ; \bullet\right)$ and $f_{2}\left(d q_{1}, d p_{1}, d q_{2}, d p_{2} ; \bullet\right)$ are equal, which proves the lemma.

Lemma 4. Let $q_{1}, p_{1}, q_{2}, p_{2}$ be integers with $1 \leq q_{1} \leq p_{1}, 1 \leq q_{2} \leq p_{2}$. If $q_{1}>q_{2}$ then $f_{1}\left(q_{1}-1\right)>f_{2}\left(q_{1}-1\right)$.

Proof.- According to our notation convention $f_{1}\left(q_{1}-1\right)$ and $f_{2}\left(q_{1}-1\right)$ stands here for $f_{1}\left(q_{1}, p_{1}, q_{2}, p_{2} ; q_{1}-1\right)$ and $f_{2}\left(q_{1}, p_{1}, q_{2}, p_{2} ; q_{1}-1\right)$.

With $n=q_{1}-1$ in function $f_{1}$, we get $a=0$ and $b=q_{1}-1$. As $b<q_{1}$, $\alpha$ and $\beta$ are such that $\alpha q_{2}+\beta=$ $q_{1}-1,0 \leq \beta<q_{2}$ and $\alpha>0$ because $q_{1}>q_{2}$. then $f_{1}\left(q_{1}-1\right)=\alpha p_{2}+\beta$.

On the other hand, in function $f_{2}$ for $n=q_{1}-1, c=\alpha$ and $d=\beta$. Then $\alpha p_{2}+\beta=\gamma p_{1}+\delta$ with $0 \leq \delta<p_{1}$.

- If $\gamma=0$, then $\delta=\alpha p_{2}+\beta>\alpha q_{2}+\beta=q_{1}-1$ and thus $f_{2}\left(q_{1}-1\right)=-\infty$.

- If $\gamma>0$ and $\delta \geq q_{1}$ then $f_{2}\left(q_{1}-1\right)=-\infty$.

- If $\gamma>0$ and $\delta<q_{1}$ then $f_{2}\left(q_{1}-1\right)=\gamma q_{1}+\delta<\gamma p_{1}+\delta=\alpha p_{2}+\beta=f_{1}\left(q_{1}-1\right)$.

Lemma 5. Let $q_{1}, p_{1}, q_{2}, p_{2}$ be integers with $1 \leq q_{1} \leq p_{1}, 1 \leq q_{2} \leq p_{2}$. If $q_{1}<q_{2}$ then $g_{1}\left(q_{2}-1\right)>g_{2}\left(q_{2}-1\right)$.

Proof.- As $q_{2}>q_{1}$, the set of parameters $q_{2}, p_{2}, q_{1}, p_{1}$ satisfy the condition given by Lemma 4 implying $f_{1}\left(q_{2}, p_{2}, q_{1}, p_{1} ; q_{2}-1\right)>f_{2}\left(q_{2}, p_{2}, q_{1}, p_{1} ; q_{2}-1\right)$.

Using Lemma 1, we get $g_{1}\left(q_{1}, p_{1}, q_{2}, p_{2} ; q_{2}-1\right)>g_{2}\left(q_{1}, p_{1}, q_{2}, p_{2} ; q_{2}-1\right)$ i.e. $g_{1}\left(q_{2}-1\right)>g_{2}\left(q_{2}-1\right)$.

Let us consider now the case of equal block lengths $q_{1}=q_{2}=q$.

Lemma 6. Let $q, p_{1}, q_{2}, p_{2}$ be integers, $1 \leq q \leq p_{2}<p_{1}$ such that $p_{1}=k q+r, p_{2}=l q+s$ with $0 \leq r<s<q$. Then $f_{1}\left(p_{1}+q-s\right)=p_{2}+q-s$ and $f_{2}\left(p_{1}+q-s\right)=-\infty$.

Proof.- In the evaluation of $f_{1}\left(p_{1}+q-s\right)$, we get $a=1, b=q-s$ since $0<q-s<q$. Now $a q+b=2 q-s$ and thus $\alpha=1$ and $\beta=q-s$.

Finally $f_{1}\left(p_{1}+q-s\right)=\alpha p_{2}+\beta=p_{2}+q-s$.

From $f_{2}\left(p_{1}+q-s\right)=f_{2}(k q+r+q-s)$, we get $c=k$ and $d=r+q-s$ since $0 \leq r \leq r+q-s<q$. Then

$$
c p_{2}+d=k(l q+s)+r+q-s=l(k q+r)+(k-1) s-(l-1) r+q,
$$

The conditions $p_{1}>p_{2}$ and $r<s$ imply that $k>l$ and thus $(k-1) s-(l-1) r+q>q$.

As $s<q,(k-1) s-(l-1) r+q<k q<p_{1}$, we get $c p_{2}+d=\gamma p_{1}+\delta$ with $\gamma=l$ and $\delta=(k-1) s-(l-1) r+q>q$. So $f_{2}\left(p_{1}+q-s\right)=-\infty$. 
Lemma 7. Let $q, p_{1}, q_{2}, p_{2}$ be integers, $1 \leq q \leq p_{2}<p_{1}$ such that $p_{1}=k q+r, p_{2}=l q+s$ with $r>0$ and $0 \leq s \leq r<q$. Then $f_{1}\left(p_{1}+q-1\right)>f_{2}\left(p_{1}+q-1\right)$.

Proof.- In the evaluation of $f_{1}\left(p_{1}+q-1\right)$, we get $a=1, b=q-1$. From $a q+b=2 q-1$, it comes $\alpha=1$ and $\beta=q-1$ and then $f_{1}\left(p_{1}+q-1\right)=p_{2}+q-1=(l+1) q+s-1$.

In the evaluation of $f_{2}\left(p_{1}+q-1\right)$, we get $p_{1}+q-1=(k+1) q+r-1$ and since $r>0, c=k+1, d=r-1$. Then $\gamma$ and $\delta$ are determined by

$$
\gamma p_{1}+\delta=c p_{2}+d=(k+1)(l q+s)+r-1,
$$

and

- If $q \leq \delta<p_{1}, f_{2}\left(p_{1}+q-1\right)=-\infty$ and the lemma is proved.

- If $0 \leq \delta<q$, first prove the inequation

$$
\gamma p_{1}+\delta<(l+1) p_{1}+s-1
$$

Using (25), we get

$$
\begin{aligned}
\Delta=(l+1) p_{1}+s-1-\left(\gamma p_{1}+\delta\right) & =(l+1)(k q+r)+s-1-((k+1)(l q+s)+r-1) \\
& =k(q-s)-l(q-r) .
\end{aligned}
$$

- If $k=l$ then $r>s$ because $p_{1}>p_{2}$, and then $\Delta=k(r-s)>0$,

- If $k>l$ and because $q-s \geq q-r>0, k(q-s)>l(q-r)$ and $\Delta>0$,

which proves that $(26)$ is satisfied.

Since the application $d\left(q, p_{1} ; \bullet\right)$ defined on $\left\{n, n \in \mathbb{Z}, \operatorname{rem}\left(n, p_{1}\right)<q\right\}$ by $d\left(q, p_{1} ; n\right)=q u o\left(n, p_{1}\right) q+$ $\operatorname{rem}\left(n, p_{1}\right)$ is a strictly increasing function, relation (26) implies

$$
f_{2}\left(p_{1}+q-1\right)=\gamma q+\delta<(l+1) q+s-1=f_{1}\left(p_{1}+q-1\right) .
$$

Proof of the theorem.- When $q_{1} \neq q_{2}$ Lemmas 4 and 5 prove that $D\left(q_{1}, p_{1}\right)$ and $E\left(q_{2}, p_{2}\right)$ cannot commute.

If $q_{1}=q_{2}=q$ and $p_{2}<p_{1}$, the only case for $(r, s)$ not considered in lemmas 6 and 7 , as shown in Figure 1, is the case where $r=s=0$, i.e. $p_{1}=k q$ and $p_{2}=l q$ with $l<k$, and thus $D\left(q_{1}, p_{1}\right)$ and $E\left(q_{2}, p_{2}\right)$ cannot commute when $p_{1}$ or $p_{2}$ are not multiples of $q$.

Using the exchange property given by Lemma 1 , we obtain a similar result for $p_{2}>p_{1}$.

So, if $q_{1}=q_{2}=q$ and $p_{1} \neq p_{2}, D\left(q_{1}, p_{1}\right)$ and $E\left(q_{2}, p_{2}\right)$ cannot commute unless $p_{1}=q p_{1}^{\prime}$ and $p_{2}=q p_{2}^{\prime}$ for some $p_{1}^{\prime}>1$ and $p_{2}^{\prime}>1$. Using Lemma 3 and Lemma 2, if $D\left(q_{1}, p_{1}\right)$ and $E\left(q_{2}, p_{2}\right)$ commute then $\operatorname{gcd}\left(p_{1}^{\prime}, p_{2}^{\prime}\right)=1$, which is equivalent to $\operatorname{gcd}\left(p_{1}, p_{2}\right)=q$.

The only case not yet considered is the case where $q_{1}=q_{2}=q$ and $p_{1}=p_{2}=p$ with $q<p$. $D(q, p)$ do not commute with $E(q, p)$ because $D(q, p) E(q, p)$ is the identity while in $E(q, p) D(q, p)$ the $x[q]$ sample is discarded, i.e. $f_{1}(q)=-\infty$ while $f_{2}(q)=q$.

This achieves the proof of the direct part of the theorem. The converse part of the theorem results immediately from Lemmas 3 and 2.

\section{REFERENCES}

[1] M. Bellanger, G. Bonnerot, and M. Coudreuse. Digital filtering by polyphase network: Application to sample rate alteration and filter banks. IEEE Trans. Acoustics, Speech, and Signal Processing, 24(2):109-114, 1976.

[2] A.A.C.M. Kalker. Commutativity of up/down sampling. Electron. Lett., 28(6):567-569, 1992.

[3] G. Cariolaro, P. Kraniauskas, and L. Vangelista. A novel general formulation of up/downsampling commutativity. IEEE Trans. Signal Processing., 53(6):2124-2134, 2005.

[4] X.-G. Xia and B.W. Suter. Multirate filter banks with block sampling. IEEE Trans. Signal Processing, 44(3):484-496, 1996. 


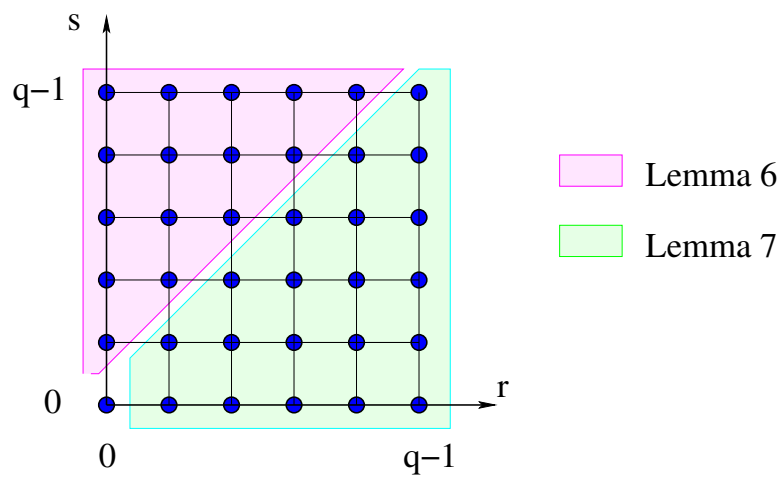

Fig. 1. Cases for $(r, s)$ studied in Lemmas 6 and 7 for $q_{1}=q_{2}=q$ and $q<p_{2}<p_{1}$.

[5] B.W.-K. Ling, C.Y.F. Ho, and Z. Cvetković. Commutativity of block decimators and expanders with arbitrary rational sampling ratios and block lengths. Digital Signal Processing, 22:677-680, 2012.

[6] C.Y.F. Ho, B.W.-K. Ling, and P.K.S. Tan. Representations of linear dual-rate system via single SISO LTI Filter, conventional sampler and block sampler. IEEE Transactions on Circuits and Systems-II: Express briefs, 55(2):168-172, 2008.

[7] P.P. Vaidyanathan. Multirate systems and filter banks. Prentice Hall, Englewood Cliffs, 1993. ISBN 0-13-605718-7.

[8] J. Kovacevic and M. Vetterli. Perfect reconstruction filter banks with rational sampling rates in one and two dimensions. In Proc. SPIE Vol. 1199 Visual Communications and Image Processing IV, Philadelphia, PA, pages 1258-1268, Nov. 1989. 